\title{
Research on Application of CGAR Algorithm in Wireless Network Routing Protocols
}

\author{
Chen Yuanyuan \\ Chongqing Industry Polytechnic College, Chongqing, China \\ E-mail: yuanyccq@yeah.net
}

\begin{abstract}
In order to reduce the transmission delay of the data packet in the wireless network, so as to improve the performance of the network, this paper proposes CGAR algorithm of the coding gain aware routing protocol. The algorithm uses the network coding gain and expected transmission count of the wireless link, provides the time needed for the node to transmit a data packet from the new flow in the wireless link, and take it as the routing metrics. This metric takes into consideration the factors such as the transmission delay, the time of the node's contention channel and so on. The experimental results show that this algorithm can reduce the transmission delay of the data packet and improve the performance of the network.
\end{abstract}

Keywords: Gain, Routing Protocol, Coding Graph, Measurement

\section{Introduction}

Network coding is a kind of emerging information transmission technology which overall improves the performance of system, and it is showed that network coding increases the amount of information transmitted singly by the network data, so as to reduce the delivery times of the groups and improve the transmission performance of the network data, such as improving the throughput and bandwidth utilization of the network, and balance the network load and so on. The data transmission in this paper is described as the forwarding process of the data group in the network through multiple-hop transmission, while the forwarding process of the data group on a link is described as the network data transmission [1]. Network coding is initially applied in the wired networks. In recent years, many researchers also applied the network coding method to the wireless network, in order to improve the throughput, robustness, security, energy efficiency and so on of the wireless network. With the rapid development of the wireless network technology in recent years, more and more people begin to use the wireless network for data communication, and the requirements of the users on the diversity of the network services and the transmission quality of the wireless communication constantly increase at the same time [2]. Thus, how to improve the data transmission performance of the wireless network, namely to ensure the safety and reliability of the network data transmission, and at the same time improve the utilization rate of the bandwidth and the energy resource of the network at the most extent has become one of the important problems of the network communication research. For this, based on the radio feature of the data transmission of the wireless link and the local information processing strategies of the node, network coding has become an important method to improve the transmission performance of network. At present, the data transmission technology based on network coding has proven to be the effective method which can be approximate to the theoretical transmission limitation of the network capacity, and it is identified as the important means to solve the problem of network transmission by the international academia and the U.S. military [3-6]. 
At present, some scholars applied the network coding in the routing protocol and have obtained some research results. In domestic, the scholars from the City University of Hong Kong, the Beijing Jiao tong University and other institutions have made important contributions to the research of network coding, which are mainly showed as follows: the presentation of the network coding thought. The network coding thought is firstly proposed by Yeung R. W [7-9], and Zhang Z. in 1998 and further developed and expanded by Ahlswede R. and others [10-13]. Katti and others put forward and implemented the chance-based network coding method (COPE) [14]; Le, Guo and others put forward the encoding conditions of the nodes in the multiple hops network, and found out the potential paths and the network coding potential opportunities between the information source nodes and the information sink nodes [15].

This paper mainly has development and innovation works in the following aspects:

(1) In order to reduce the data packet transmission delay of the wireless network to improve the network performance, this paper puts forward the CGAR algorithm of the coding gain aware routing protocol. The algorithm uses the network coding gain and expected transmission count of the wireless link, provides the time needed for the node to transmit a data packet from new stream in the wireless link, and take it as the routing metrics to achieve the goal of reducing the time delay. This paper provides the greedy algorithm of the coding gain and the simplified method of the coding graph.

(2) To further verify the correctness and effectiveness of the CGAR algorithm proposed in this paper, the simulation experiment compared with COPE and DCAR protocols is carried out, and experiment adopts the MATLAB to carry out the simulation experiment. Randomly generate 25 nodes in the range of $100 \mathrm{~m} \times 100 \mathrm{~m}$, the communication range of each node is $35 \mathrm{~m}$, and the length of the explorer packet is L0 $=137 \mathrm{~B}$. In the aspect of time delay, CGAR is better than DCAR and COPE. The time delay increases with the increasing of the data packet's success rate of transmission. And the path's failure rate of CGAR is lower than that of DCAR and COPE. The results of the simulation experiment show that the algorithm in this paper can reduces the transmission delay of the data packet and improve the performance of the network.

\section{Network Coding and Gain}

In the network topology graph which is shown in Figure 1, assume that the bandwidth of the network link is $2 \mathrm{M}$ bps, there exists the data flow if $(i=1,2,3)$, if transfers from node 1 to node 10 at a speed of $1.5 \mathrm{Mbps}$, $2 \mathrm{f}$ transfers from node 1 to node 9 at a speed of $0.5 \mathrm{Mbps}$, and $3 \mathrm{f}$ transfers from node 2 to node 9 at a speed of $2 \mathrm{M}$ bps. Figure 2 is the model diagram where $2 \mathrm{f}$ and $3 \mathrm{f}$ realize the rate matching coding of data in coding node 6 , in the figure $23 \mathrm{f}$ is the encoding data flow. The figure shows that because $1 \mathrm{f}$ and $3 \mathrm{f}$ have the matched data transmission rate, and the data rate, and the queue length are the statistically significant parameters, aiming at the single queue, the data rate describes the arrival rate of the queue data, and the forwarding rate of encoding describes the data service rate of the queue. In the network coding, the corresponding encoding forwarding rates of the data groups of the different data flows after encoding are the same, therefore, the corresponding queue length of each data flow depends on the arrival rate of data. And under the condition that different data flows have the matched data rates, the corresponding queue length of the encoding data are equal in the statistical sense, therefore, in the process of encoding of $1 \mathrm{f}$ and $3 \mathrm{f}$ in node 6 , there exist the equal queue length, which creates conditions for the complete coding between the two kinds of data flows. 


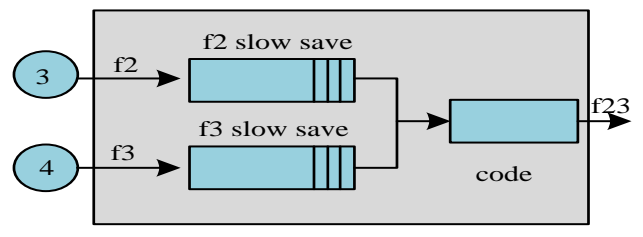

Figure 1. The Flows Enter into Node $X$

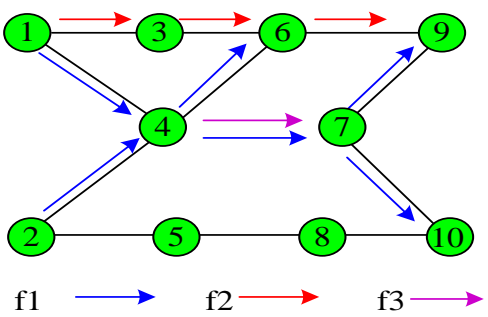

Figure 2. The Coding Graph G (V, E) of Node X

The coding graph of each node can be constructed by using the above "coding conditions". In the coding graph, if a certain sub graph of a graph meets the condition that there exists an edge between any two vertexes which can connect the two vertexes together, then the sub graph is a "complete sub graph". All the flows that constitute a complete sub graph can be encoded at the same time. As shown in Figure 2, flow $f_{1}, f_{2}$ and $f_{3}$ constitute a complete sub graph of coding graph $\mathrm{G}$, there is a connection between every two vertices.

In the process of network coding, the improving of coding opportunities shows that the amount of data transmission reduces, and the reducing of the amount of data transmission reduces the cost of network data transmission, and obtains the improving of coding gains. Under the condition that the network topology is stable, for the network coding between the convections, the coding level of data in the coding node directly affects the size of the network coding opportunities, and it depends on the data rate matching state of the data flow in the coding node in a large extent. Based on this, the paper discusses the establishing of the relationship between the data rate and the amount of data transmission, and then establishes the cost efficiency function of the network based on the data rate, so as to describe the change of the network coding gain. Based on this, the paper adopts the linear programming model of the correlation data rate to solve the data rate matching problem in network coding. The specific description is as follows: set that the rate of data flow $f_{i}$ is $x_{i}$, and the transfer rate of $f_{i}$ on path $y$ is $x_{i}^{y}$, and it meets the following formula: $\sum_{y \in y_{i}} x_{i}^{y}=x_{i}\left(x_{i}^{y} \in x_{i}, x_{i} \in x, x=\left[x_{1}, x_{2}, \ldots x_{F}\right]\right)$, $y_{i}$ is the route set passed by $f_{i}$, and $F$ is the set of the network data flow, the success transfer rate of data on link $\left(n_{i}, n_{j}\right)$ is $a_{i j},\left(a_{i j}\right)$, define the probability that node $n_{j}$, successfully receives the data groups of node $n_{i}$, and it is the size of cost of transmission on $\left(n_{i}, n_{j}\right)$ in the paper. For path $y\left(n_{i}, n_{i}, n_{j}\right)$ and path $y\left(n_{i}, n_{i}, n_{j}\right) n_{k}$ is the coding node, after introducing the network encoding mechanism, the transmission costs produced by the data flows transferred with $x_{i}^{y}, x_{j}^{y}$ on path $\mathrm{p}$ and $\mathrm{q}$ are as follows:

$$
\begin{aligned}
u_{n_{i}}\left(x_{i}, y_{i}\right) & =\max \left\{\alpha_{i j}, a_{k i}\right\} y_{i} \\
& =x_{n_{i}} x_{i}^{y}+x_{n_{i}} x_{j}^{y}-\left[\left(\alpha_{i j}+a_{k i}\right)-\max \left(\alpha_{i j}, a_{k i}\right) y_{i}\right]
\end{aligned}
$$


In formula (1), there is $u_{k}=\min \left\{x_{i}^{y}, x_{j}^{y}, y_{i}\right\}, y_{i}$ the capacity of path $\mathrm{p}$, and $y_{i}$ is the vector of the network link state, namely the capacity of link, and then the transmission cost of the network system is described as follows:

$$
u(x, y)=\sum_{f_{i} \in F_{x}} \sum_{x \in x_{i}}\left(\theta_{i}^{x} y_{j}^{r}-R\right)
$$

In formula (2), there is $u_{k}=\min \left\{x_{i}^{y}, x_{j}^{y}, y_{i}\right\}, y_{i}, \theta_{i}^{y}$ and $C N_{p} f_{i}$ is respectively the transmission cost of $i_{j}$ vector and the set of encoding nodes of $f_{i}$ on path $\mathrm{p}$. At the same time, the required amount if transfer rate of the non-coding data and coding data of if on the route of $c_{n}$ is less than the currently available capacity $x_{y}$ of the path, namely $\sum_{x \in x_{i}} x_{i}^{y} \leq y_{c}$, and it can be known from (2) that to make the transmission cost of the network data obtains the minimum value, the matching rate of network data coding should be obtained the maximum value under the condition of meeting the capacity limit of the link, namely be expressed as the following linear programming model:

$$
\max \sum_{f_{i} \in F_{x} \in x_{i}} T
$$

\section{The CGAR Algorithm}

\section{A. The Routing Metric}

After using the network coding, in one new flow that through node $i$, part of the flow can be encoded with the existing flows without taking up the additional channel capacities. This means that when sending the data packet of the new flow, the available channel capacity of node $i$ is increased by the encoding gain $\triangle A S(K)$ on the basis of the original unoccupied channel capacity. That is to say, after using the network coding, the available channel capacity when node $i$ is ending the data packet of the new flow is as follows:

$$
S(K)=\Delta A S(K)+S_{i}(K)
$$

Set $\mathrm{L}$ for the length of the data packet sent by the node, and its unit is bit. As a result, with the support of network coding, the time needed for transmitting a data packet from the new flow in the channel of node $i$ is L/C (i), and its unit is s. Use ETX $(i)$ to show the number of transmission needed for the node to successfully transmit a data packet from the new flow. In this way, the average time needed for the node to transmit a data packet from the new flow is as follows:

$$
\begin{aligned}
\Phi(i) & \left.=\left\lfloor(\operatorname{ETX}(i)-1)\left(\frac{M}{y(i)}+t_{0}\right)\right]\right\rfloor \\
& +\left\lceil\frac{M}{y(i)}+\delta+T_{0}\right\rceil+\operatorname{ETX}(i) T_{y}
\end{aligned}
$$

Among which, $t_{0}$ is the value of the timer set up by node $i$ to the sent frame, $\delta$ is the propagation delay, TACK is the transmission delay of the ACK packet, and TC is the time consumed when the node is competing the channel. In the first line of formula (4), the item in the square bracket shows the time consumed when node $i$ fails to send the data packet in the former ETX $(i)-1$ times and it needs to retransmit the data packet in the timeout of the timer. In the second line, the item in the square bracket shows the time consumed when node $i$ successfully receiving the confirmation 
packet, namely, the ACK packet, in the ETX (i) time of sending the data packet. In the second line, the last item shows the total time consumed when node $i$ is in the ETX (i) time of competing the channel.

In the routing algorithm of this paper, when node $i$ is carrying out the routing, Error! Reference source not found. $(i)$ is treated as the routing metric, in which, the parameters $M, E T X(i)$ and C (I) are calculated by the following methods.

(1) The calculation of $M$.

In the standard of IEEE 802.31, the type of frame is divided into three categories, the data frame, the management frame and the control frame, in which the maximum length of frame is 2401 bytes (the data frame) and the minimum length of frame is 34 bytes (the control frame). Assume that the size of the frame is in accordance with the uniform distribution, then its average value is $(34+2346) / 2=1190$ (Byte).

(2) The calculation of ETX (i) .

ETX $(i)$ is the average time of the transmission when node $i$ successfully send a data packet, and ETX(i) is related to the packet loss rate of the node's data transmission. De Couto and others use the method of radio probe packet to obtain the approximation of the packet loss rate of the node's data transmission, when the data length of the probe packet is $M_{0}$ bytes. And the approximation is as follows:

$$
y=1-\left(1-y_{x}\right)\left(1-y_{u}\right)
$$

Among which, $y_{x}$ and $y_{u}$ are respectively the packet loss rates of the forward sent probe packet and the reverse sent confirmation package. Therefore, for the data packet with a length of $M$ bytes, it can be divided into $M / M_{0}$ data packets with a length of $M_{0}$. Then, the success probability of transmitting a data packet with a length of $L$ bytes is $\left(1-y_{u}\right) M / M_{0}$, and the packet loss rate is as follows:

$$
y_{M}=1-\left(1-y_{u}\right)^{M / M_{0}}
$$

So, the average transmission number for node $i$ to transfer a data packet with a length of $M$ bytes is as follows:

$$
E T X_{M}=\frac{1}{1-y_{M}}=\frac{1}{(1-y)^{M / M_{0}}}
$$

As mentioned earlier, the value range of $M$ is in line with the uniform distribution of 35 2401, so the expectation of ETXL is as follows:

$$
E T X(i)=E\left[E T X_{M}\right]=\frac{1}{2401-35} \int_{35}^{2401} \frac{1}{(1-y)^{M / M_{0}}}
$$

Through the calculation of formula (9), the obtained formula is as follows:

$$
\operatorname{ETX}(i)=\frac{M_{0}(1-y)^{-35 / M_{0}}-(1-y)}{2401(1-y)}
$$

(3) The calculation of $H(i)$.

When a new flow with a flow of $\mathrm{Q}$ is passing node $i$, its obtained new network coding gain within $\Delta T$ time can be calculated by using the following formula: 


$$
\begin{array}{r}
\Delta A S(K)=A S_{\text {new }}(i)-A S_{\text {old }}(i) \\
=\frac{I F_{\text {oL }}(i)+u-O F_{\text {new }}}{\Delta T}
\end{array}
$$

Among which, $I F_{\text {old }}(i)$ and $A S_{\text {old }}(i)$ are respectively the input flow and output flow of node $i$ before the entering of the new flow, $I F_{\text {old }}(i)$ and $O F_{n e w}(i)$ respectively shows the input flow and output flow of node $i$ after the entering of the new flow and its participate in the coding.

It can be known from formula (3) that the expand channel capacity $u(i)$ of node $i$ is proportional to $\triangle A S(K)$. Therefore, it is necessary to analyze the effects of the changes of the new flow's flow $u$ on the new coding gain $\triangle A S(K)$. When there is $u^{\prec} I F_{\text {old }}(i)$, namely, the flow of the new flow is less than the existing flow that pass node $i$, part of the flow of the new flow can be tool away by the existing flow through the coding, and with the increasing of $\mathrm{Q}$, the difference between $O F_{\text {new }}(i)$ and $I F_{\text {old }}(i)$ is not big. Therefore, it can be known from formula (10) that $\triangle A S(K)$ increases with the increasing of Q. However, when there is $u \succ I F_{\text {old }}(i)$, the flow $u-I F_{n e w}(i)$ cannot be encoded with the existing flow and be took away, and $O F_{\text {new }}(i)$ increases with the increasing of $\mathrm{Q}$, which makes $u-O F_{n e w}(i)$ remain the same, it can be known by formula (10) that $\triangle A S(K)$ almost remains the same. That is to say, under the condition of $u \succ I F_{\text {old }}(i)$, increase the input flow $u$ cannot produce new coding gain.

Based on the above consideration, when calculating the coding gain brought by the new flow of node $i$, there is $u=I F_{\text {old }}(i)$ is this paper, in which, $I F_{\text {old }}(i)$ shows the sum of the flow of the existing flow when passing node $i$, except for the new flow. In this way, the obtained formula through formula (3) and (10) is as follows:

$$
u(i)=\frac{I F_{\text {old }}(i)-\left[I F_{\text {old }}(i)-O F_{\text {new }}(i)\right]}{\Delta T}+u_{1}(i)
$$

\section{B. The Route Discovery}

The routing protocol of CGAR is similar to that of AODV. The path-finding process is initiated by the source node, who broadcasts the routing request packet RREQ (Route Request) to the neighbor node, the intermediate node forwards RREQ to the neighbor node, and the information sink (the destination node) returns RREP(Route Reply) to the information source after receiving RREQ, after the information source receives RREP, it chooses a path. When there are multiple paths between the information source and the information sink to be chosen from, the information source will the path with the minimum sum of Error! Reference source not found.(i) of each node in the path.

RREQ contains the domains such as the making ReqID of RREQ packet, the current node identifier NID, the information source SRC, the information sink DST, the set of the upstream nodes UpSet, namely, the collection of the nodes from the information source in the flow to the current node's path, the hop neighbor set of the upstream nodes UpNbSet and so on. RREP contains the domains such as the making RepID of RREP packet, the ReqID, the current node identifier NID, the information source SRC, the information sink DST, the transmission delay of the path, the set of the downstream nodes DnSet, namely, the collection of the nodes from the current node in the flow to the information sink's path, the hop neighbor set of the downstream nodes DnNbSet.

Each node is deployed with the "path-finding table" and the "flow chart", which respectively record the information used in the path-finding process and the information of the existing flow in the node. The former contains the domains such as the ReqID, 
RepID, SRC, DST, UpSet, UpNbSet, DnSet, DnNb - Set, NextNode To SRC (the next hop node from the node to the information source), NextNode to DST (the next hop node from the node to the information sink), Valid-Time, which shows the survival time of the corresponding record in the table, and the unit is ms. The later contains the domains such as the FID (identifier of the flow), SRC, DST, UpSet, UpNbSet, DnSet, DnNbSet, Q ( the flow passed within $\Delta t$ time, and the unit is Mb), NextHopTo SRC ( the next hop node to the information source), NextHop to DST ( the next hop node to the information sink). For example, for node 3 in Figure 4, its flow chart is shown in table 1 , in which the value of $\mathrm{Q}$ is refreshed every $\Delta \mathrm{t}$ time.

Table 1. The Flow Chart

\begin{tabular}{|l|l|l|l|l|l|}
\hline NextHopTo SRC & UpSet & DnNbSet & UpNbSet & ReqID & DnSet \\
\hline 4 & 2 & 8 & 2 & 9 & 5 \\
\hline 8 & 5 & 5 & 6 & 3 & 6 \\
\hline
\end{tabular}

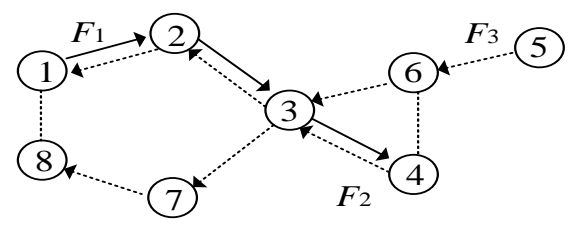

Figure 1. The Flows through Node 3

The route discovery process of CGAR protocol is as follows:

The source node broadcasts RREQ to the neighbor node, in which ReqID is the unique identifier of RREQ packet, NID and SRC both are the numbers of the source node, DST is the number of the destination node, and UpSet and UpNbSe are empty. At the same time, add a record (namely add a line) in the path-finding table of the destination node, and fill the above contents in the corresponding field.

After the intermediate node receives the RREQ packet, handle the RREQ package according to the following logic:

Step 1 if in the received RREQ, the triple (SRC, DST, ReqID) is the same with the certain record in the path-finding table of the node, then abandon RREQ and go to Step 7.

Step 2 if the bigram (SRC, DST) in RREQ is the same with the corresponding content recorded in the path-finding table, go to step 3, otherwise, go to the Step4.

Step 3 if the domain of UpSet in the path-finding table is the subset of the UpSet in RREQ, then abandon this RREQ packet and go to Step7; otherwise, delete the corresponding record in the path-finding table, and go to Step4.

Step 4 add a new record in the path-finding table, copy the domains ReqID, SRC, DST, UpSet and UpNbSet into the path-finding table, and copy the RREQ.NID into the NextNode To SRC,FID domain in the path-finding table, the content will be automatically generated, and increased by 1 every time.

Step 5 modifies the RREQ packet. Modify the NID domain into the number of the node, add the number of the node to UpSet, and add the next hop node of the node to the UpNbSet domain.

Step 6 broadcast the RREQ packet to the neighbor node.

Step 7 END.

After the information sink receives the RREQ packet, carry out the managements as follows:

Step 1 if the UpSet recorded in the path-finding table is the subset of RREQ. UpSet domain, then abandon the RREQ packet, and go to Step4, otherwise, go to Step2. 
Step 2 add a record in the path-finding table, copy the data in the ReqID, SRC, DST, UpSet, UpNbSet domain in the RREQ into the record to produce the only identification RepID of RREP and put it into the path-finding table to automatically generate FID, DnSet domain and DnNbSe domain both are empty, and copy the RREQ.NID into the NextNode To SRC of the path-finding table.

Step 3 produce RREP, in which domain $\Phi$ is initialized to 0 , copy the content of the path-finding table into the corresponding domain of RREP, and single-cast RREP to the referred node of domain NextNode To SRC in the path-finding table.

Step 4 ENDS.

After the intermediate node (node i) receives the RREP packet, carry out the managements as follows:

Step 1 initialize Old-GF=New-GF=0, among which, $I F_{\text {old }}(i)$ and $I F_{\text {new }}(i)$ both are the memory variables that are respectively used to record the old coding gain and new coding gain of the node.

Step 2 according to the triple (SRC, DST, ReqID), find the corresponding record in the path-finding table, and fill the DnSet, DnNbSet domain of RREP into it.

Step3 if there is no flow in the flow chart, then take the extended channel capacity $y_{(i)}=y_{i}$, go to the Step 6.

Step 4 calculates the sum of the flow of all the flows in the flow chart, namely the value of domain $\mathrm{Q}$.

Step 5 use the content of UpSet and DnSet in the path-finding table and the flow chart, and determine whether if the encoding can be carried out according to the "coding conditions". If the encoding can be carried out, set Old-GF=New-GF, calculate the coding gain $A S(i)$ according to the former "greedy coding gain algorithm", and set $N e w-A S=A S(i)$.

Step 6 make use of $I F_{\text {old }}(i), I F_{\text {new }}(i)$, calculate the new coding gain $\triangle A S(K)$ according to formula (10).

Step 7 calculate ETX (i), according to formula (9), and calculate Error! Reference source not found.(i) according to formula (4), fill it into the $\Phi$ domain of the path-finding table, and accumulate it to the RREP domain.

Step 8 adds the node to the RREP. DnSet domain, add the hop neighbor node of this node to RREP. DnNbSet domain, and copy RREP.NID to the NextNode to DST of the path-finding table.

Step 9 is to single-cast RREP to the referred node of the domain NextNode to SRC, in the path-finding table.

Step 10 Ends.

After the information source receives RREP, a path to the information sink is obtained. If the information source receives multiple RREP packets with the same triple (SRC, DST, ReqID), then the information source will choose the path with the minimum value of Error! Reference source not found. to send the data packet. When each node in the chosen path receives the actual transmission packet, add a record in the flow chart and delete all the records in the path-finding table.

\section{Experimental Simulation and Analysis}

\section{A. The Experimental Settings}

Use the design language of the Matlab programming to compile the simulation program to carry out the experiment, and analyze the experimental results. In figure 1, the numbers next to the link show the distances between the nodes. The same as the literature [9], the loss index $y$ of the path is 2, and there are Eelec $=52 \mathrm{~nJ} / \mathrm{bit}$ and Eamp $=12 \mathrm{pj} / \mathrm{bit} / \mathrm{m} 2$. When solving the combinatorial 
optimization problem (17), the CGAR algorithm described in Section 3 is used, and the bytes occupied by the header of the data packet and the ACK packet both are 30 bytes, namely, $v=m=30$ bytes. Realize the throughput and delay of the three routing algorithms, CGAR, DCAR, and COPE. The simulated experiment scenario is shown in Figure 4 .

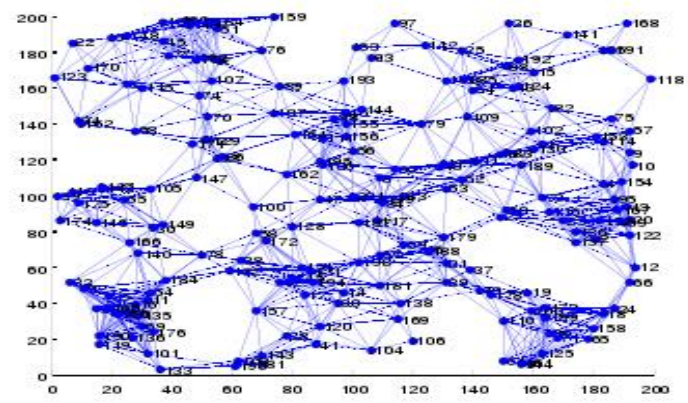

Figure 1. The Simulation Diagram

\section{B. The Analysis of the Results}

Respectively in view of the condition that there are $1,2, \ldots 10$ flows (randomly select a pair of nodes to form the information source and the information sink of a flow) in the network and the condition of the change of the flow number (hereinafter referred to as "flow"), the simulation results of the throughput and the delay of the three algorithms CGAR, DCAR and COPE are shown in Figure 5 and Figure 5.
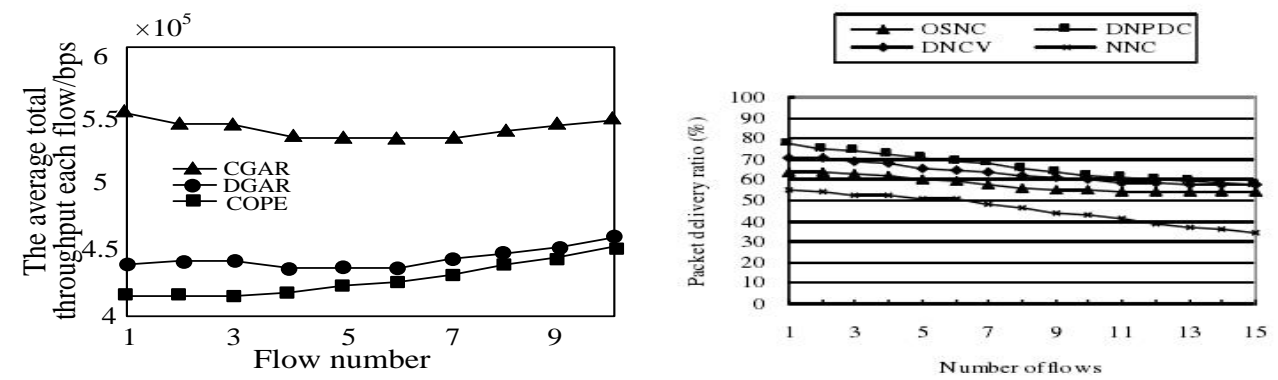

Figure 1. The Average Though Put of Each Flow
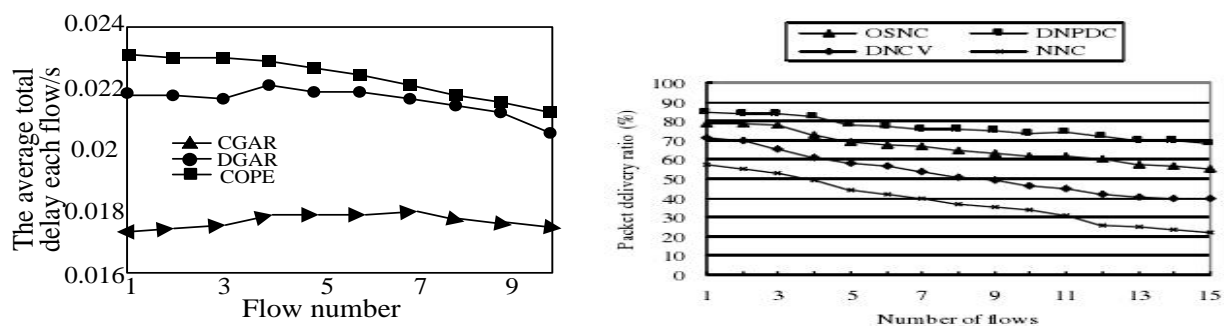

Figure 2. The Average Delay of Each Flow

It can be seen from the two figures that, (1) for the given number of flow, CGAR is better than DCAR and COPE in terms of throughput and delay; (2) when the number of flow is relatively small in the network, the delay of CGAR is on the rise with the increasing of the number of the flow. But when the number of the flow increases to a certain degree, the delay gradually shows a trend of declining, this is mainly because with the increasing of the number of the flow, the node can encode the data packets 
from multiple flows to generate a coding packet, so as to improve the efficiency and save time.

Let the success rate of the probe packet changes between $0.90 \sim 1.03$, namely the packet loss rate changes between $0 \sim 0,18$, the simulation results of the changes of the delay can be obtained, and it are shown in Figure 7. This shows that: (1) CGAR is still better than DCAR and COPE in the aspect of delay; (2) the delay of CGAR declines with the increasing of the success rate of the data packet, this is because the success rate is large, the number of the retransmission of the data packet declines and the delay reduces accordingly.

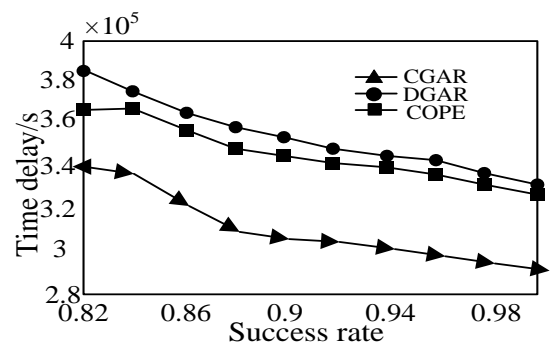

\section{Figure 3. The Relationship between Success Rate and Delay}

Under the condition that the number of the flow changes, the simulation results of the path failure rate of the CGAR, DCAR and COPE routing are shown in Figure 8. The so-called path failure refers to that there is node in the path which cannot send the data packet due to the lack of channel capacity. In addition, the path failure rate is defined as the proportion of the number of the failed paths in the total number of the paths determined by the routing algorithm. Figure 8 shows that: (1) for the given number of the flow, the path failure rate of CGAR is lower than that of DCAR and COPE; (2) with the increasing of the number of the flow, the path failure rate of the three types of routing protocols increases, which is in line with the intuitive. Because the load of the each path's transmission of the data packet increases with the increasing of the number of the flow, as so to make the lack of bandwidth, which leads to the failure of the path.

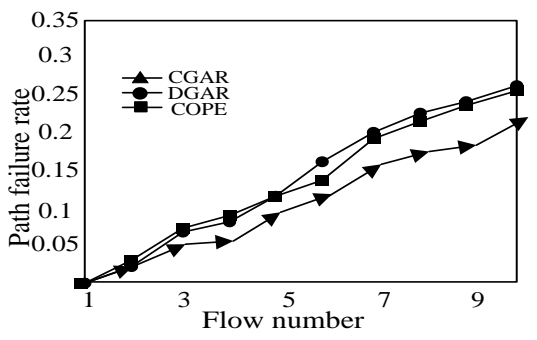

Figure 4. The Path Failure

\section{Conclusion}

Routing protocol is a hot research topic in the current wireless sensor network, this paper studies the application of the proposed CGAR algorithm in the wireless network routing protocol. The algorithm can effectively reduce the energy consumption, relieve the radio interference between the nodes, save the transmission delay of the node used in retransmitting the cache of the needed configuration of the data packet and the data packet, and reduce the loss rate of the path, so as to improve the reliability of data transmission in the process of coding. To further explore the effects of the distribution of coding nodes and the configuration of the network data flow model on the data 
transmission delay during coding, and the time synchronization problem of the nodes during coding, it remains to be further implementation in the future research.

\section{Acknowledgment}

This research work is supported by Chongqing Board of Education Science and technology research project (KJ1403001).

\section{References}

[1] G. Tan, C. Sun and P. Wang, “Interaction Design of National Dance Based on Realistic 3D Character”, Journal of Multimedia, vol. 8, no. 4, (2013), pp. 338-344.

[2] A. Hilton, D. Beresford, T. Gentils, et al., "Virtual people: capturing human models to populate virtual worlds, Computer Animation, (1999), pp. 174-185.

[3] V. Atanasovski, V. Rakovic and L. Gavrilovska, "Efficient resource management in future heterogeneous wireless networks: The RIWCoS approach," Military Communications Conference, vol. 7, (2010), pp. 2286-2291.

[4] O. Fratu, A. C. Boucouvalas, L. Gavrilovska, et al, " RIWCoS: Possible approach for the reconfiguration of the hybrid wireless access networks, 2011 2nd International Conference on Wireless Communication", Vehicular Technology, Information Theory and Aerospace \& Electronic Systems Technology (Wireless VITAE), (2011), pp. 1-5.

[5] J. Perez-Romero, O. Salient, R. Agust, "Policy-based initial RAT selection algorithms in heterogeneous networks", In International Workshop on Mobile and Wireless Communications Network (MWCN), (2005).

[6] F. Yu and V. Krishnamurthy, "Optimal joint session admission control in integrated WLAN and CDMA cellular networks with vertical handoff”, IEEE Transactions on Mobile Computing, vol. 6, no. 1, (2007), pp. 126-139.

[7] A. Farbod and B. Liang, "Structured Admission Control Policy in Heterogeneous Wireless Networks with Mesh Underlay", IEEE INFOCOM, (2009), pp. 495-503.

[8] L. Zhihan, L. Feng, H. Li, and S. Feng, "Hand-free motion interaction on Google Glass", In SIGGRAPH Asia 2014 Mobile Graphics and Interactive Applications, (2014), p. 21, ACM.

[9] Z. Chen, S. M. Arisona, X. Huang, M. Batty, and G. Schmitt, "Detecting the dynamics of urban structure through spatial network analysis", International Journal of Geographical Information Science, vol. 28, no. 11, (2014), pp. 2178-2199.

[10] L. Wubin, J. Tordsson, and E. Elmroth, "An aspect-oriented approach to consistency-preserving caching and compression of web service response messages", In Web Services (ICWS), 2010 IEEE International Conference on, (2010), pp. 526-533.

[11] S. Li, Y. Geng, J. He and K. Pahlavan, "Analysis of Three-dimensional Maximum Likelihood Algorithm for Capsule Endoscopy Localization", 2012 5th International Conference on Biomedical Engineering and Informatics (BMEI), Chongqing, China, (2012) October, pp. 721-725.

[12] Y. Geng, J. He, H. Deng and K. Pahlavan, "Modeling the Effect of Human Body on TOA Ranging for Indoor Human Tracking with Wrist Mounted Sensor", 16th International Symposium on Wireless Personal Multimedia Communications (WPMC), Atlantic City, NJ, (2013) June.

[13] D. Niyato and E. Hossain, "Radio resource management games in wireless networks: an approach to bandwidth allocation and admission control for polling service in IEEE 802.16", IEEE Wireless Communications, vol. 14, no. 1, (2007), pp. 27-35.

[14] L. Zhihan, L. Feng, S. Feng, and H. Li, "Extending Touch-less Interaction on Vision Based Wearable Device." Virtual Reality (VR), 2015 iEEE. IEEE, (2015).

[15] Z. Mengxin, Z. Lv, X. Zhang, G. Chen, and K. Zhang, "Research and Application of the 3D Virtual Community Based on WEBVR and RIA", Computer and Information Science 2, no. 1, (2009), p. 84.

[16] D. Niyato and E. Hossain, "A cooperative game framework for bandwidth allocation in 4G heterogeneous wireless networks", ICC, vol. 6, no. 9, (2006), pp.4357-4362.

\section{Authors}

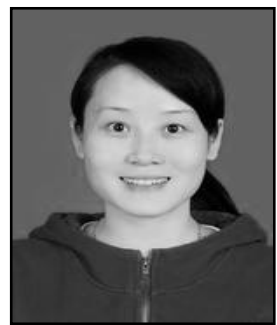

Chen Yuanyuan, was born in 1982. She is a lecture of Chongqing Industry Polytechnic College. Her research interest is mainly in the area of Computer Software, Mechanical and Electrical Integration. She has published several research papers in scholarly journals in the above research areas and has participated in several books. 
International Journal of Future Generation Communication and Networking Vol. 8, No. 2 (2015) 